\title{
Soft Palate Carcinoma
}

National Cancer Institute

\section{Source}

National Cancer Institute. Soft Palate Carcinoma. NCI Thesaurus. Code C8395.

A carcinoma that arises from the soft palate. The majority are squamous cell carcinomas. 